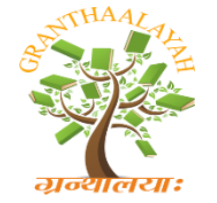

$$
\begin{gathered}
\text { INTERNATIONAL JOURNAL OF RESEARCH - } \\
\text { GRANTHAALAYAH } \\
\text { A knowledge Repository }
\end{gathered}
$$

RACEEE - 17

\title{
INDOOR LOCALIZATION SOLUTION FOR GPS
}

\author{
Shreyanka B. Chougule ${ }^{1}$, Dr.Sayed Abdulhayan ${ }^{2}$ \\ ${ }^{1}$ Electronics \&Communication Department, Dayananda Sagar College of Engineering, \\ Bangalore, India \\ ${ }^{2}$ Telecommunication Department, Dayananda Sagar College of Engineering, Bangalore, India
}

DOI: https://doi.org/10.29121/granthaalayah.v5.i4RACEEE.2017.3334

\begin{abstract}
GPS technology is used for positioning application and it is highly reliable and accurate when used outdoor. Due to multipath propagation, signal attenuation and blockage its performance is limited in indoor and dense urban environment. As a solution, technologies like Apple's iBeacon, Radio-frequency identification (RFID), Ultrasonic and Wireless Fidelity (Wi-Fi) access points are used to improve performance in Indoor environment. We are having a look at all these technologies which are meant for GPS Indoor performance improvement in this review paper.
\end{abstract}

Keywords: GPS; Wi-Fi; GPS Repeaters; RFID.

Cite This Article: Shreyanka B. Chougule, and Dr.Sayed Abdulhayan. (2017). "INDOOR LOCALIZATION SOLUTION FOR GPS." International Journal of Research - Granthaalayah, 5(4) RACEEE, 124-129. https://doi.org/10.29121/granthaalayah.v5.i4RACEEE.2017.3334.

\section{Introduction}

The GPS allows 3D outdoor positioning where the receiver is in visibility with at least four satellites (three satellites for 2D-positioning). Although this system is operational outdoors, its use inside buildings is too limited. Indeed, in an indoor environment, GPS signals are weakened by walls and other obstacles, which make the detection very delicate or even impossible with a GPS receiver. We use a number of technologies to recover from Indoor localization some of which are wireless technologies like ibeacon, RFID and Wi-Fi etc. Besides the use of wireless transmission technologies, other types of sensors such as the gyroscope, accelerometer and compass found in wearable devices or Smartphone's as well as laser technology are also applied in recent indoor localization investigations. Many solutions will depend on a longer preparation and learning phase before the systems can be ready to be deployed. The alternative to the above techniques will be the solutions that leverage on any existing infrastructure. Such solutions will incur lower costs since no new installation of hardware is required. For example, Wi-Fi infrastructure is very common nowadays for many existing buildings. If a feasible solution can 
Recent Advances in Communication, Electronics \& Electrical Engineering be built based on Wi-Fi technology, it will be seen as a cost effective solution as compared to those mentioned above.

This paper is arranged into sections as Introduction, section-2 as Different technologies speaking about the solution to indoor localization problem, section-3 as ibeacon description, section-4 as sensor depicting use of sensors, odometer and GNSS Repeater for solving indoor localization problem,section-5 as Bluetooth beacon depicting its employment as solution,section-6 speaks about Wi-Fi access point usage, section-7 conveys the results and section 8 conveys conclusion of this review paper.

\section{Different Technologies}

Indoor localization systems are using available Global Positioning System (GPS) signals and simple deployment of so-called "GPS repeaters." The delay-lock loop (DLL) architecture of a GPS receiver has been modified into an open DLL to improve indoor positioning performance. Some of these techniques make use of radio technology that serves as hooks or sensors for indoor positioning. Firstly explanation on technologies like Apple's iBeacon, Radio-frequency identification (RFID), Ultrasonic and Wireless Fidelity (Wi-Fi) access points is given.

\section{Apple iBeacon}

Apple iBeacon is one of the cases of indoor situating, where it utilizes Bluetooth reference points to ping for the area of a client from a specific signal to appraise the region of the client. An organization named Wifarer knows about the open air - just GPS framework, and has thought of a framework that utilizations Wi-Fi Access Points and Bluetooth Low Energy reference points to create advanced fingerprints to position the clients indoor. Notwithstanding, both techniques utilize the Bluetooth LE signal and Wi-Fi Access Points for situating. Such methodologies will require the clients to put resources into new foundations. Reference points are commonly transmitters in customer based techniques. For instance, they empower indoor route for aircraft travelers utilizing the application - cross-stage and with an exactness of up to 1 meter. The server-based reference point following of people or products is just conceivable with outsider parts (e.g. Cisco, Aruba).

When you utilize guides for indoor route, impermanent establishments, e.g. for displays and in irregular areas are additionally conceivable. Mounting the Beacons is basic and to a great degree adaptable. The lodging is accessible in various hues with the goal that gadgets can be introduced circumspectly. The Bluetooth Low Energy (BLE) innovation empowers battery operation more than two to eight years without accessing an outer power supply. Reference points can likewise be associated with the power supply or utilize the power supply from the lighting. Paper-thin reference points with a printed battery are perfect for exceptionally prudent and space-sparing establishments, for instance, on ISO cards or on promoting materials. They have a battery life of three to four days 
Recent Advances in Communication, Electronics \& Electrical Engineering

\section{Sensors, Odometer, GNSS Repeater}

Sensors, for example, the compass, spinner, gauge, accelerometer, pneumatic stress and attractive fields, with the assistance of Wi-Fi, GPS (for open air), Bluetooth and 3G/4G association. However, this strategy requires extra foundation, where serious site overview of finding the extra Bluetooth sensors must be accomplished for the entire framework to run, which will take quite a while and it is additionally expensive with respect to labor and time, not to overlook the span of the building.

The odometer, which is a standard segment in Antilock Braking Systems (ABS) considered as a wheel speed sensor, is utilized as a part of request to give a consistent route, when the GPS measures are not accessible. A worldwide demonstrating of the GPS/INS/Odometer combination issue, with long GPS blackouts. This multisensor estimation issue is tackled by a Kaiman channel that wires the estimations of every sensor to gauge position, speed and state of mind of the vehicle.

A GNSS repeater is much the same as a wireless repeater that is utilized to help the mobile phone gathering by utilizing a gathering receiving wire, a flag intensifier and an inward rebroadcast radio wire. Rather than cell communicate stations, GNSS repeaters are considerably littler and regularly introduced inside structures with an outer gathering radio wire gathering the satellite signs. By introducing a GNSS repeater, we can get live GNSS flags even in indoor situations. In any case, as is outstanding, the indoor position arrangement decided with a GNSS collector utilizing a repeater flag is really the area of the open air gathering reception apparatus in light of the fact that the GNSS repeater acts like a link interfacing outside radio wire and indoor recipient. In this way, the additional way deferral (through the repeater) is normal to all satellites in view, and is along these lines vague from the beneficiary clock counterbalance. The fundamental thought basic the utilization of repeaters is to disentangle the requirement for extra framework by utilizing genuine GNSS signals. Specifically, open air GNSS signs are increased, exchanged, and additionally deferred so as to have the capacity to figure a position inside. Pseudolites, interestingly, have their own pseudo-clamor (PN) codes like those transmitted by the GNSS satellites.

The way to utilizing a GNSS repeater for situating is a radio recurrence (RF) exchanging gadget. This gadget takes a solitary info (i.e., the live flags from outside) and switches it among different re-radiation receiving wires, each one in turn. By methods for this time area multiplexing, beneficiaries can successively track GNSS signals from the various re-radiation radio wires introduced at various areas without self-impedance. With this exchanging among the re-radiation radio wires a change happens in the flag retransmission way. The change relates to the time distinction of landing (TDOA) between the exchanged retransmission receiving wires and the client. In this way, in the event that we have four retransmission receiving wires associated with the exchanging repeater, we can acquire three TDOA estimations for three-dimensional situating. In the event that the utilization of GNSS repeater is took into consideration certain indoor conditions under RF radiation controls, exchanging and additionally postponing GNSS signals empowers indoor situating, with no new PN code and flag generator as with pseudolites. 
Recent Advances in Communication, Electronics \& Electrical Engineering

\section{Bluetooth Beacons}

Bluetooth guides are little radio transmitters that convey motions in a range of 10-30 meters (inside spaces). The benefits of signals are self-evident: They are financially savvy (three to thirty euros), can be introduced with negligible exertion, decide a position precisely up to 1 meter and are upheld by many working frameworks and gadgets. The new BLE (Bluetooth Low Energy) standard is likewise exceptionally vitality proficient. Reference points can be utilized for both customer based and also server-based applications. With signals it is conceivable to recognize the present floor.

Bluetooth Low Energy Beacons are accessible from various merchants and come in different shapes and sizes for various utilize cases. Utilizing the business standard Bluetooth Smart, the arrangements of infsoft are perfect with reference points of all producers. infsoft does not create signals itself, but rather we are glad to place you in contact with relating suppliers.

Bluetooth reference points ordinarily don't influence other radio systems (obstruction) and they likewise don't meddle with medicinal gadgets. Be that as it may, in the event that you introduce guides in a space with heaps of Wi-Fi signals (for instance, at an expo), then impedance can happen in light of the fact that BLE and Wi-Fi have a similar recurrence run $(2.4 \mathrm{GHz})$. The issue can be effectively evaded here by not utilizing channels $2,3,4,13$ and 14 while arranging the Wi-Fi and utilizing 1, 6, 7, 8, 9, 10,11 and 12. Bluetooth utilizes the staying accessible diverts to limit in a uniform way (recurrence jumping). Publicizing channels that are utilized for situating are set apart in yellow in the realistic. The blue-shaded channels are saved for extra capacities, for example, a temperature sensor.

There are lessenings in the flag scattering inside structures for BLE reference points. Relating attributes are considered when introducing and amid parameterization.

- Low constriction properties: Wood, manufactured materials, glass

- Medium constriction properties: Brick, marble

- High lessening properties: Plaster, concrete, covered glass surfaces (impenetrable renditions, and so forth.)

- To a great degree high lessening properties: Metal, water (this incorporates individuals and gatherings of individuals)

Indoor situating with Ultra-wideband has some critical points of interest: The precision is 10-30 $\mathrm{cm}$, which is impressively superior to anything when working with guides (1-3 meters) or Wi-Fi (5-15 meters). Dormancy time is low (position ask for up to 100 circumstances/second). Stature contrasts can be measured precisely. Be that as it may, the strategy is an exceptional arrangement which requires fitting segments and hence is generally reasonable for extraordinary industry applications.

Ultra-wideband is a short-go radio innovation which can be utilized for indoor situating. As opposed to Bluetooth Low Energy and Wi-Fi, situating is finished with travel time approach (Time of Flight, ToF) rather than the estimation of flag qualities (Receive Signal Strength 
Recent Advances in Communication, Electronics \& Electrical Engineering

Indicator, RSSI). This technique measures the running time of light between a protest and a few beneficiaries (Anchors - infsoft Locator Nodes). For the correct confinement of a question no less than 3 beneficiaries are vital (trilateration).

The protest or the individual that ought to be followed (resource) is outfitted with a little tag (infsoft Locator Tag) which keeps running on battery control. It sends information (ID, ToF, timestamp) to the infsoft Locator Nodes. They have a settled position in the foundation and can utilize the running time of light to compute the separation of the advantage. Consolidating the information of 3 Locator Nodes or more outcomes in a situating exactness of $10-30 \mathrm{~cm}$.

\section{Specialized Features of Ultra-wideband}

- Usage of to a great degree wide recurrence groups with a data transfer capacity of no less than $500 \mathrm{MHz}$

- Almost no impedances

- Frequency transmission capacity $3.1-10.6 \mathrm{GHz}$

- Transmission control $0.5 \mathrm{~mW} /-41.3 \mathrm{dBm} / \mathrm{MHz}$

- Reach $10-150 \mathrm{~m}$ (relies on upon the utilization case)

- Information rate $110 \mathrm{kbit} / \mathrm{s}-6.8 \mathrm{mbit} / \mathrm{s}$

\section{Wi-Fi Access Point}

Wi-Fi gets to point, regardless of whether client hotspot, switch or Internet-proficient purpose of offer framework, transmits particular information. Utilizing a RSSI (Received Signal Strength Indication) and MAC address (Media Access Control), an application can ascertain the present area of the end client gadget (customer based situating). This requires a database with data about the areas with which this information can be looked at. This strategy is called fingerprinting. It just capacities with Android gadgets because of specialized confinements. IoS gadgets can't be utilized for Wi-Fi indoor route.

Alternately, the information from all Wi-Fi able gadgets (free of the producer) or Wi-Fi labels from infsoft Locator Nodes can be recorded for server-based situating (e.g. for resource following or course examination) and sent to the server.

The exactness of Wi-Fi for indoor situating is commonly 5-15 meters since get to focuses are typically utilized whose position has been enhanced for information correspondence. This accuracy relies on upon the protecting through dividers, roofs and individuals, and additionally the quantity of get to focuses. The utilization of Smartphone sensors can enhance the outcomes and the assurance of the floor level is additionally conceivable.

\section{Results and Discussions}

Many of the above technologies require new installations of dedicated sensors and incur additional costs and resources. It will be particularly costly when such solutions should be implemented in existing buildings. For example, iBeacon or technology based on Bluetooth Low 
Recent Advances in Communication, Electronics \& Electrical Engineering

Energy will have limited range and may require extensive calibration if high accuracy on the variety of spaces is required. An RFID-based solution will require sufficient base stations to be installed in designated areas and dedicated RFID tags needed to be worn. Then the solution is we have to work with the existing technologies. For example, Wi-Fi infrastructure is very common nowadays for many existing buildings. If a feasible solution can be built based on Wi-Fi technology, it will be seen as a cost effective solution as compared to those mentioned.

\section{Conclusions \& Recommendations}

Hence by referring to all the technologies mentioned we can conclude the we need to Implement Indoor localization solution by fusing already existing Wi-Fi access point with that of GPS one.

\section{Acknowledgements}

The Research on Topic of GPS fusion with Wi-Fi access point is being carried out at Dayananda Sagar College of Engineering R\&D centre as part of mini project work by Shreyanka B. Chougule under the guidance of Dr.Sayed Abdulhayan.

\section{References}

[1] Nabil jardak, Nelsamama"Indoor Positioning Based on GPS-Repeaters: Performance Enhancement using an Open Code Loop Architecture", IEEE transactions on aerospace and electronic systems vol. 45, no. 1 january 2009.

[2] Jee, G.-I., Lee, J.-H., and Im, S.-H. "Indoor positioning using time synchronized switching GPS repeater" Presented at ION GNSS 2005, Long Beach, CA.

[3] Vervisch-Picois, A., Bideau, A., Jeannot, M., and Samama, N. 2D-indoor dynamic positioning using GNSS based repeaters. Presented at ION GNSS 2006, Fort Worth, TX, Sept. 2006.

[4] Indoor Positioning Based on Global Positioning System Signals Kerem Ozsoy, Ayhan Bozkurt and Ibrahim Tekin Electronics Engineering, Sabanci University 34956, Istanbul, Turkey.

[5] https://www.infsoft.com/technology/sensors/bluetooth-low-energy-beacons

*Corresponding author.

E-mail address: chouguleshreyanka@gmail.com 